\title{
A colonic mass revealing a disseminated crystal storing histiocytosis secondary to indolent multiple myeloma: a case report with literature review
}

Adrien Contejean ${ }^{1,2}$, Frédérique Larousserie ${ }^{2,3}$, Didier Bouscary ${ }^{1,2}$, Anthony Dohan ${ }^{2,4}$, Bénédicte Deau-Fischer ${ }^{1}$, Tali-Anne Szwebel ${ }^{5}$, Marion Dhooge ${ }^{6}$, Benoit Terris ${ }^{2,3}$ and Marguerite Vignon ${ }^{1,2^{*}}$

\begin{abstract}
Background: Crystal storing histiocytosis is a rare disorder associated with monoclonal gammopathy. In this disease, monoclonal heavy and light chains accumulate in the lysosome of macrophages, leading to histiocytic reaction in different organs. It is secondary to the presence of a small B-cell clone, responsible for monoclonal immunoglobulin production. Histological diagnosis is a challenge and differential diagnoses include fibroblastic and histiocytic neoplasm. Clinical manifestations depend on the involved organs, rarely including peritoneum or digestive tract.

Case presentation: We present a case of a 75-year-old with a medical history of colonic carcinoma. She presented with abdominal pain and inflammatory syndrome revealing a colonic mass. Hemicolectomy was performed. Initial diagnosis was fibroblastic tumour. The patient worsened, and diagnosis of a diffuse crystal storing histiocytosis was finally done. Haematological exploration found an indolent lgG-kappa multiple myeloma. The initial treatment with conventional chemotherapy did not permit an improvement of the patient condition. Immunotherapy with anti-CD38 monoclonal antibody (daratumumab) was proposed with a clinical and biological response.

Conclusion: This case report emphasizes the histopathological challenge of histiocytic tumours which may involve digestive track. It focuses on the concept of monoclonal gammopathy of clinical significance, which can have a large spectrum of manifestations.
\end{abstract}

Keywords: Cristal storing histiocytosis, Multiple myeloma, Daratumumab, Case report

\footnotetext{
* Correspondence: marguerite.vignon@aphp.fr

'Haematology Department, AP-HP, Cochin University Hospital, 27 rue du

Faubourg Saint Jacques, Paris, France

${ }^{2}$ Paris University, Paris, France

Full list of author information is available at the end of the article
}

(C) The Author(s). 2020 Open Access This article is licensed under a Creative Commons Attribution 4.0 International License, which permits use, sharing, adaptation, distribution and reproduction in any medium or format, as long as you give appropriate credit to the original author(s) and the source, provide a link to the Creative Commons licence, and indicate if changes were made. The images or other third party material in this article are included in the article's Creative Commons licence, unless indicated otherwise in a credit line to the material. If material is not included in the article's Creative Commons licence and your intended use is not permitted by statutory regulation or exceeds the permitted use, you will need to obtain permission directly from the copyright holder. To view a copy of this licence, visit http://creativecommons.org/licenses/by/4.0/ The Creative Commons Public Domain Dedication waiver (http://creativecommons.org/publicdomain/zero/1.0/) applies to the data made available in this article, unless otherwise stated in a credit line to the data. 


\section{Background}

Crystal storing histiocytosis $(\mathrm{CSH})$ is a rare disorder associated with monoclonal gammopathy. It is defined by accumulation of monoclonal heavy and light chains in the lysosome of macrophages. $\mathrm{CSH}$ may result in a variety of clinical manifestations depending on the involved organs, but digestive tract involvement is rare.

\section{Case presentation}

A 75-year-old woman presented with abdominal pain and altered general condition. Her medical history was left colectomy for localised colonic adenocarcinoma 12 years ago, radiotherapy for anal epidermoid carcinoma diagnosed on a histologic piece of hemorrhoidectomy 3 years ago, type II diabetes, and IgG-kappa monoclonal gammopathy. She reported an abdominal discomfort for more than 1 year then weight loss in the past 6 months. These symptoms were explored a year ago with CT-scan and colonoscopy that were normal. At admission, CTscan showed a pseudotumoural thickening of the right colonic wall with diffuse peritoneal effusion (Fig. 1A) but the colonoscopy without biopsies revealed no mucosal abnormalities. A surgical exploration was decided: peroperative examination revealed a mass infiltrating the right colon, the caecum, and the peritoneum leading the surgeon to perform a right hemicolectomy. First histological conclusion (Fig. 1B) was desmoid-type abdominal fibromatosis and after review fibroblastic/myofibroblastic tumour of intermediate malignancy. No treatment was proposed, and the patient was addressed to our institution.

The patient's condition worsened within 6 months with a loss of $12 \mathrm{~kg}$, diarrhoea, abdominal pain a slowing down of her intestinal tract and dysphagia, without vomiting. Clinical examination showed a sub-mandibular tumefaction which was confirmed on a CT-scan (Fig. 1C). Abdominal imaging showed peritoneal effusion and multiples nodules suggesting carcinosis. Biopsy of the submandibular mass showed numerous histiocytes with intracytoplasmic eosinophilic crystals, engulfed within a dense reactional fibroblastic population (Fig. 1D). Those findings allowed a diagnosis of $\mathrm{CSH}$. Immunohistochemistry confirmed macrophagic nature of the cell infiltrate,

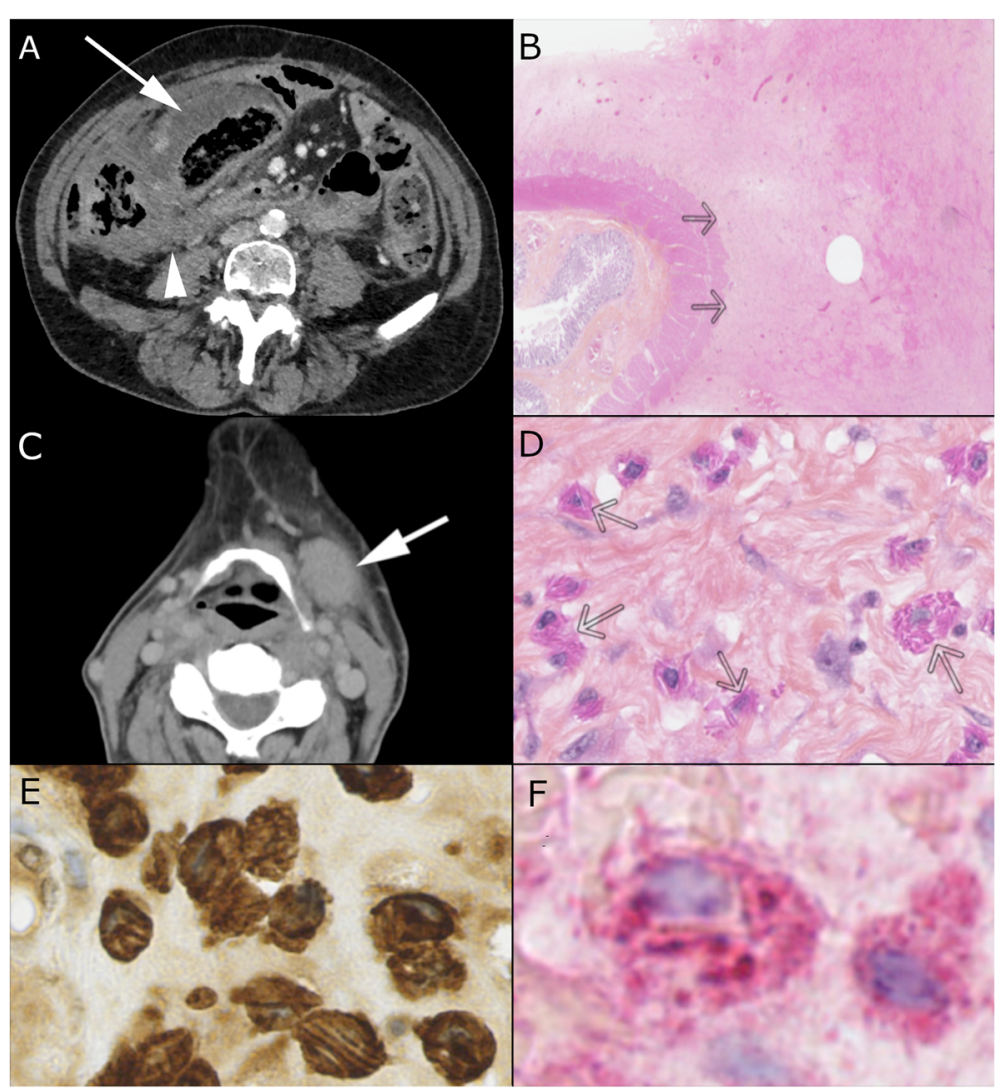

Fig. 1 CT-scan and histological analyses. a: Axial section of abdominal CT-scan showing a pseudotumoral thickening of the right colonic wall (arrow) with diffuse peritoneal effusion (arrowhead). b: Right colectomy showing a tumoral proliferation developed from subserosal part of the bowel without infiltration of muscularis. c: Axial section of a cervical CT-scan showing a left sub-mandibular lymphadenopathy of 30 mm in diameter (arrow). d: The tumour comprised numerous histiocytes with crystals in eosinophilic cytoplasm (arrows). e: Histiocytes are positive for CD163. f: Crystals are positive for the kappa light chain 
positive for CD163 (Fig. 1E). Crystals were stained with an anti-kappa antibody (Fig. 1F) and not with an anti-lambda antibody, consistent with a monoclonal light chain. The review of the colonic mass histology confirmed the diagnosis and allowed us to classify it as a generalized $\mathrm{CSH}[1]$.

Haematological explorations detected a monoclonal IgG-kappa at $26 \mathrm{~g} / \mathrm{l}$ and imbalanced kappa/lambda free light chains (Tables 1). Serum creatinine and calcium were normal. Haemoglobin level was $10.8 \mathrm{~g} / \mathrm{dL}$. A bone marrow aspiration found $16 \%$ of dysmorphic plasma cells. Cytogenetics identified a $t(11 ; 14)$ translocation. PET-scan was normal. A diagnosis of indolent IgG-kappa multiple myeloma (MM) complicated with diffuse CSH was made. A first line treatment based on proteasome inhibitor was proposed with bortezomib (1.3 mg/m2 D1;D4;D8;D11), cyclophosphamide $(350 \mathrm{mg} / \mathrm{m} 2 \quad \mathrm{D} 1 ; \mathrm{D} 8 ; \mathrm{D} 15)$ and dexamethasone (40 mg D1-D4). After three cycles, the general status of the patient worsened with persistent abdominal pain and a marked inflammatory syndrome. Introduction of continuous corticosteroid therapy with prednisone at $0.8 \mathrm{mg} / \mathrm{kg} / \mathrm{d}$ improved the abdominal pain.

A second-line regimen based on immunodulatory agents and monoclonal antibody was done, with daratumumab (1400 mg), lenalidomide (25 mg D1-D21) and dexamethasone ( $40 \mathrm{mg} /$ week). Partial response (PR), defined by reduction of $50 \%$ of gammopathy level, was obtained (Table 1) with significant improvement of the patient's condition. Although abdominal CT-scan showed persistence of peritoneal nodules, we noticed a disappearance of peritoneal effusion. After 6 months of treatment, immunochemical PR persisted and albumin normalized (Table 1). Medullar biopsy was normal. Unfortunately, 2 months later a mechanical occlusion of the intestine with perforation occurred. The evolution was rapidly fatal with multiple organ failure syndrome and death of the patient despite intensive care and surgical management.

\section{Discussion and conclusions}

Monoclonal gammopathies always result from B-cell clones and can be related to MM or lympho-plasmocytic lymphoma. Sometimes the B-clone is quiescent, but organ damage can occur due to the toxicity of the monoclonal immunoglobulin itself, or by other mechanisms. Thus the concept of monoclonal gammopathy with clinical significance (MGCS) was introduced [2]. Most MGCS-associated lesions are caused by the deposition of entire or parts of the monoclonal immunoglobulins. Crystalline deposits are present in three distinct entities: acquired Fanconi syndrome, crystalline keratopathy and CSH. We must make a distinction between localized $\mathrm{CSH}$, involving one organ system, often in the head and neck region (35\%) and diffuse $\mathrm{CSH}$, involving two or more distant organ sites [1]: bone marrow (97\%), liver (47\%), spleen (44\%) and lymph nodes (44\%) which are the most frequent. Digestive tract involvement is rare. Inflammatory syndrome may occur during generalized $\mathrm{CSH}$.

In $\mathrm{CSH}$, light chain is almost always kappa, suggesting that occurrence of $\mathrm{CSH}$ is mainly linked to structural characteristics of the monoclonal immunoglobulin. Plasma cells produce a structurally aberrant immunoglobulin which aggregates in crystals accumulated in the lysosome of macrophages because of proteolysis resistance [3]. The mechanism that promotes crystallization of protein and that affects intra-lysosomal degradation remains unclear.

The diagnosis of CSH represents a clinical and histopathological challenge, especially in peritoneal and digestive tract involvement where peritoneal carcinosis may be wrongly suggested. Characterization of histiocytes with abundant crystalline inclusions is the main feature of CSH [4]. Cytologically benign histiocytes contain eosinophilic crystals that distend their cytoplasm. Immunohistochemistry demonstrates intra-cytoplasmic inclusions made of monotypic light and/or heavy chains of immunoglobulins. There are numerous differential diagnoses of histiocytic reaction. In our case a diagnosis of fibroblastic tumour was initially done.

In a review, 23 cases of generalized CSH among a total of $131 \mathrm{CSH}$ cases were identified [5]. Their prognosis is worse because of organ impairment. As in other MGCS,

Table 1 Biological results at baseline and under treatment

\begin{tabular}{|c|c|c|c|c|}
\hline & Diagnosis & $\begin{array}{l}\text { After C3 } \\
\text { Bor-Cyc-Dex }\end{array}$ & $\begin{array}{l}\text { After C3 } \\
\text { Dara-Len-Dex }\end{array}$ & $\begin{array}{l}\text { After C6 } \\
\text { Dara-Len-Dex }\end{array}$ \\
\hline Monoclonal spike (g/L) & 26 & 23 & 5 & 3.3 \\
\hline Free light chains kappa/lambda (mg/L) & $54 / 7$ & $107 / 3$ & $29 / 1$ & $27.1 / 1.4$ \\
\hline Hemoglobin (g/dL) & 10.8 & 10.3 & 12 & 11.8 \\
\hline Serum calcium (mmol/L) & 2.3 & 2.03 & 2.21 & 2.33 \\
\hline Serum creatinine $(\mu \mathrm{mol} / \mathrm{L})$ & 73 & 75 & 88 & 85 \\
\hline Albumin (g/L) & 27 & 26 & 30 & 40 \\
\hline CRP $(\mathrm{mmol} / \mathrm{L})$ & 85 & 106 & 2 & 1 \\
\hline
\end{tabular}

Bor-Cyc-Dex Bortezomib Cyclophosphamide Dexamethasone; CRP C-Reactive Protein; Dara-Len-Dex Daratumumab Lenalidomide Dexamethasone 


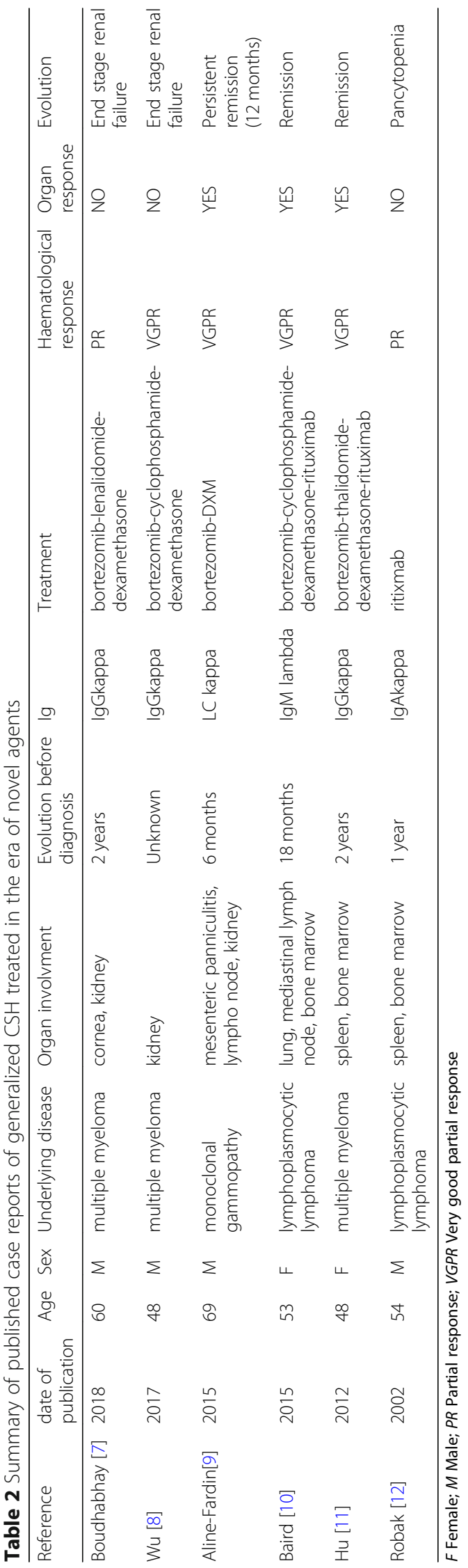


treatments recommendation is to target the underlying malignancy to stop the production of the monoclonal immunoglobulin [6]. However, despite haematological response, the clearing of histiocytic lesions is inconsistent. Between 2000 and 2019, six detailed cases of generalized $\mathrm{CSH}$ treated in the era of novel agents have been published (Table 2). CSH was diffuse and involved kidney $(n=2)$, bone marrow and spleen $(n=2)$, mesenteric panniculitis $(n=1)$, and lung $(n=1)$. Three patients had a MM $[2,3,13]$ two a lymphoplasmacytic lymphoma $[5,6]$ and one monoclonal gammopathy [7]. Light chain was always kappa (IgG [3], IgA [1], IgM [1] or LC only [1]). Bortezomib base therapy was used in five of them and rituximab in one case. Four patients obtained a very good partial response and two patients obtained a PR. In 3/6 cases haematological response did not stop organ impairment and patients evolved to end stage renal failure $(n=$ $2)$ or pancytopenia $(n=1)$. In one case patient general status improved but mesenteric lymph nodes were still present. Two cases had a complete organ response.

Here, we describe the first case of a patient with $\mathrm{CSH}$ treated with daratumumab-based therapy. Daratumumab is a novel targeted anti-CD38 monoclonal antibody that is being increasingly used in the treatment of MM. In a relapse setting, the association of daratumumab with lenalidomide and dexamethasone permits an overall response rate of $92 \%$ in patients with MM [13]. In the context of AL amyloidosis, daratumumab can be used in frail patients with promising results [14]. Thus, immunotherapy in the management of MGCS seems to have an increasing role by improving the control of toxic immunoglobulin production. A better molecular understanding of disease may help to define the optimal treatment.

\section{Abbreviations}

CSH: Crystal Storing Histiocytosis; MGCS: Monoclonal Gammopathy with

Clinical Significance; MM: Multiple Myeloma; PR: Partial Response

\section{Acknowledgments}

Not applicable.

\section{Authors' contributions}

$A C, F L, D B, A D, M D, B T$ and $M V$ participated to the writing of the manuscript. $A C$ and MV were responsible for data collection and literature review. FL and BT performed the histological analyses. AD performed the imaging analyses. BDF, TAS and MD participated to the patient's management. All authors read and approved the final manuscript.

\section{Funding}

Not applicable.

\section{Availability of data and materials}

Not applicable.

Ethics approval and consent to participate

Not applicable.

\section{Consent for publication}

The patient provided a non-opposition statement. After her death, her daughter gave us a written consent to publication.

\section{Competing interests}

All authors declare no conflict of interest.

\section{Author details}

${ }^{1}$ Haematology Department, AP-HP, Cochin University Hospital, 27 rue du Faubourg Saint Jacques, Paris, France. ${ }^{2}$ Paris University, Paris, France.

${ }^{3}$ Pathology Department, AP-HP, Cochin University Hospital, Paris, France. ${ }^{4}$ Radiology A Department, AP-HP, Cochin University Hospital, Paris, France. ${ }^{5}$ Internal medicine Department, AP-HP, Cochin University Hospital, Paris, France. ${ }^{6}$ Gastroenterology Department, AP-HP, Cochin University Hospital, Paris, France.

Received: 19 December 2019 Accepted: 2 July 2020

Published online: 31 July 2020

References

1. Dogan S, Barnes L, Cruz-Vetrano WP. Crystal-storing Histiocytosis: report of a case, review of the literature (80 cases) and a proposed classification. Head Neck Pathology. 2012;6(1):111-20.

2. Fermand J-P, Bridoux F, Dispenzieri A, Jaccard A, Kyle RA, Leung N, et al. Monoclonal gammopathy of clinical significance: a novel concept with therapeutic implications. Blood. 2018;132(14):1478-85.

3. Lebeau A, Zeindl-Eberhart E, Müller E-C, Müller-Höcker J, Jungblut PR, Emmerich B, et al. Generalized crystal-storing histiocytosis associated with monoclonal gammopathy: molecular analysis of a disorder with rapid clinical course and review of the literature. Blood. 2002;100(5):1817-27.

4. Kanagal-Shamanna R, Xu-Monette ZY, Miranda RN, Dogan A, Zou D, Luthra $R$, et al. Crystal-storing histiocytosis: a clinicopathological study of 13 cases. Histopathology. 2016;68(4):482-91.

5. Galeano-Valle F, Díaz-Crespo FJ, Melero-Martín R, Apaza-Chávez JE, Del-ToroCervera J, Demelo-Rodríguez P. Massive generalized crystal-storing histiocytosis associated with extracellular crystalline nephropathy: clinical, immunohistochemical, and ultrastructural studies of a unique disorder and review of the literature. CEN Case Rep. 2019;2.

6. Fermand J-P, Bridoux F, Kyle RA, Kastritis E, Weiss BM, Cook MA, et al. How I treat monoclonal gammopathy of renal significance (MGRS). Blood. 2013; 122(22):3583-90.

7. Boudhabhay I, Titah C, Talbot A, Harel S, Verine J, Touchard G, et al. Multiple myeloma with crystal-storing histiocytosis, crystalline podocytopathy, and light chain proximal tubulopathy, revealed by retinal abnormalities. Medicine (Baltimore). 2018;97(52) [cited 2019 Apr 29] Available from: https:// www.ncbi.nlm.nih.gov/pmc/articles/PMC6314660/.

8. Wu C-K, Yang A-H, Lai H-C, Lin B-S. Combined proximal tubulopathy, crystalstoring histiocytosis, and cast nephropathy in a patient with light chain multiple myeloma. BMC Nephrol. 2017;18(1):170.

9. Aline-Fardin A, Bender S, Fabiani B, Buob D, Brahimi S, Verpont MC, et al. Pseudo-peritoneal Carcinomatosis presentation of a crystal-storing Histiocytosis with an Unmutated monoclonal $\mathrm{k}$ light chain. Medicine (Baltimore). 2015;94(32):e1247.

10. Baird SM, Kenealy MK, Hoy R. Complete remission of Waldenström's associated generalized crystal-storing histiocytosis of IgM lambda subtype with bortezomibbased combination chemotherapy. Leuk Lymphoma. 2015;56(11):3233-5.

11. Hu X, Liu J, Bai C, Wang J, Song X. Bortezomib combined with thalidomide and dexamethasone is effective for patient with crystal-storing histiocytosis associated with monoclonal gammopathy of undermined significance. Eur J Haematol. 2012;89(2):183-4.

12. Robak T, Urbańska-Ryś H, Jerzmanowski P, Bartkowiak J, Liberski P, Kordek R. Lymphoplasmacytic lymphoma with monoclonal gammopathy-related pseudo-Gaucher cell infiltration in bone marrow and spleen--diagnostic and therapeutic dilemmas. Leuk Lymphoma. 2002;43(12):2343-50.

13. Dimopoulos MA, San-Miguel J, Belch A, White D, Benboubker L, Cook $G$, et al. Daratumumab plus lenalidomide and dexamethasone versus lenalidomide and dexamethasone in relapsed or refractory multiple myeloma: updated analysis of POLLUX. Haematologica. 2018.

14. Abeykoon JP, Zanwar S, Dispenzieri A, Gertz MA, Leung N, Kourelis T, et al. Daratumumab-based therapy in patients with heavily-pretreated AL amyloidosis. Leukemia. 2019;33(2):531-6.

\section{Publisher's Note}

Springer Nature remains neutral with regard to jurisdictional claims in published maps and institutional affiliations. 